\title{
Evaluation of left ventricular dyssynchrony using combined pulsed wave and tissue Doppler imaging
}

Xuedong Shen ${ }^{1}$, Wilbert S. Aronow ${ }^{2}$, Kishlay Anand ${ }^{1}$, Chandra K. Nair', Mark J. Holmberg', Tom Hee', Stephanie Maciejewski ${ }^{1}$, Dennis J. Esterbrooks ${ }^{1}$

${ }^{1}$ Cardiac Center of Creighton, University School of Medicine, Omaha, USA ${ }^{2}$ Cardiology Division, New York Medical College, Valhalla, USA

Submitted: 22 October 2008

Accepted: 28 December 2008

Arch Med Sci 2010; 6, 4: 519-525

DOI: 10.5114/aoms.2010.14462

Copyright $\odot 2010$ Termedia \& Banach

\section{Abstract}

Introduction: The combination of pulsed wave (PW) and tissue Doppler imaging (TDI) has been proposed as a new method to assess left ventricular (LV) mechanical dyssynchrony (LVMD), but results have not been validated. We investigated the correlation of a combination of PW and TDI with a positive response to cardiac resynchronization therapy (CRT).

Material and methods: We studied 108 consecutive patients who received CRT. Patients with atrial fibrillation were excluded. The time difference $\left(T_{P W-T D I}\right)$ between onset of QRS to the end of LV ejection by PW (TPW) and onset of QRS to the end of the systolic wave in LV basal segments with greatest delay by TDI (T TDI $)$ was measured before CRT and during short-term and long-term follow-up.

Results: The $\mathrm{T}_{\mathrm{PW} \text {-TDI }}$ interval before CRT was $74 \pm 48 \mathrm{~ms}$. Intra-observer variabilities for $T_{P W}$ and $T_{T D I}$ were $1.5 \pm 0.24 \%$ and $1 \pm 0.17 \%$. Inter-observer variabilities for $T_{P W}$ and $T_{T D I}$ were $1 \pm 0.36 \%$ and $1 \pm 0.64 \%$, respectively. $T_{P W-T D I}>50$ ms was defined as the cutoff value for diagnosis of LVMD by receiver operating curve (ROC) analysis. During follow-up of $15 \pm 11$ months, the sensitivity and specificity of $\mathrm{TP}_{\mathrm{PW} \text {-TDI }}$ to predict a positive response to CRT were $98 \%$ and $82 \%$, respectively. The area under the ROC curve was 0.92. There was a significant agreement between LVMD determined by $\mathrm{T}_{\mathrm{PW} \text {-TDI }}$ and the positive response to CRT $(\kappa=0.80)$.

Conclusions: Left vertricular dyssynchrony detected by the method combining PW and TDI demonstrated a high reproducibility, sensitivity, specificity and agreement with a positive response to CRT.

Key words: cardiac resynchronization therapy, left ventricular mechanical dyssynchrony.

\section{Introduction}

Cardiac resynchronization therapy (CRT) is an effective treatment for patients with New York Heart Association (NYHA) class III or IV heart failure, reduced left ventricular ejection fraction (LVEF), and widened QRS duration by electrocardiogram (ECG) [1, 2]. Left ventricular mechanical dyssynchrony (LVMD) has emerged as a therapeutic target in heart failure patients with NYHA class III or IV and substantial left ventricular dysfunction [3-5]. Tissue Doppler imaging (TDI) is one of numerous methods to assess LVMD. Currently, there is no agreement as to which methods are superior to others to assess LVMD [6]. Tissue Doppler imaging assesses the absolute and relative left ventricular wall velocity and thereby quantifies the time
Corresponding author: Wilbert S. Aronow, MD, FACC, FAHA Cardiology Division New York Medical College Macy Pavilion, Room 138 Valhalla, NY 10595, USA Phone: 9144935311

Fax: 9142356274 E-mail:wsaronow@aol.com 
delay between opposing segments of the left ventricle, but results are inconsistent [7]. Left ventricular mechanical dyssynchrony determined by a method using combined pulsed wave (PW) and TDI is a novel method and has been recently reported $[8,9]$.

The aim of the current study was to evaluate the reproducibility, sensitivity, specificity, and positive and negative predictive values of this combined method in predicting a positive response to CRT.

\section{Material and methods}

We retrospectively evaluated 108 consecutive patients who received CRT (78 men and 30 women, mean age $70 \pm 10$ years) at The Cardiac Center of Creighton University School of Medicine. The criteria for bi-ventricular pacemaker implantation were the American College of Cardiology/American Heart Association 2005 guidelines [10]: 1) left ventricular ejection fraction (LVEF) $\leq 35 \%$, 2) NYHA class III-IV, 3) QRS duration (QRSD) $\geq 120 \mathrm{~ms}$, and 4) optimal pharmaceutical regimen established prior to CRT. QRSD in all patients was measured from the surface ECGs using the widest QRS complex in leads II, V1 and V6 on the surface ECG. Patients with atrial fibrillation were excluded. There were 69 patients (64\%) with ischemic cardiomyopathy (33 patients with previous myocardial infarction and 36 patients with angiographic severe coronary artery disease without myocardial infarction) and 39 patients with non-ischemic cardiomyopathy. All 108 patients underwent coronary angiography before CRT.

After informed written consent was obtained, all 108 patients underwent implantation of a biventricular pacer in the cardiac electrophysiology laboratory. The left ventricular lead was positioned

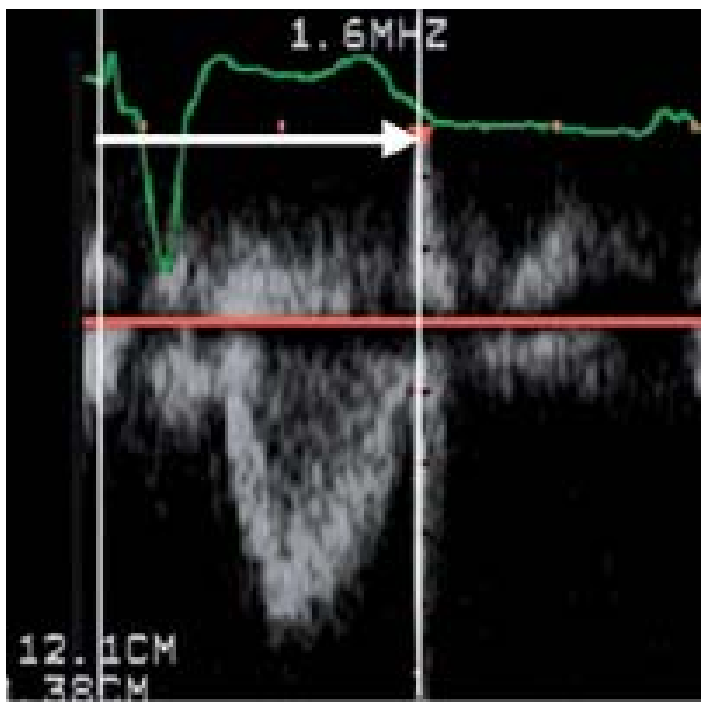

Figure 1. The time from onset of the QRS wave to the end of left ventricular ejection assessed by pulsed wave Doppler into the lateral left ventricular vein. The right ventricular defibrillation lead was actively fixated into the RV apex. The atrial pacing lead was fixated into the high lateral right atrium. All patients had successful implantation of the left ventricular lead in the lateral left ventricular vein.

Conventional transthoracic echocardiography was performed with a Philips Sonos 7500 echocardiographic system and s3 transducer. Baseline echocardiographic data before CRT and follow-up echocardiography after CRT were reviewed for all patients. End-systolic and end-diastolic left ventricular volumes were measured in the apical view from the videotape or Philips EnConcert digital system according to the standard recommended by the American Society of Echocardiography [11]. The LVEF was calculated from the apical fourchamber view of the left ventricle using Simpson's rule. A positive response to CRT was defined as the left ventricular end-systolic volume decreasing $\geq 15 \%$ after CRT [12].

Left ventricular ejection was evaluated by PW at the level of the LV outflow tract from the apical fivechamber view. Left ventricular wall motion was assessed by TDI from the four-chamber view. The following two time intervals were measured for evaluation of LVMD: measurement 1 - the onset of Q wave to the end of left ventricular ejection assessed by PW ( $\left.T_{P W}\right)$ (Figure 1); and measurement 2 - the onset of $\mathrm{Q}$ wave to the end of the systolic wave in the basal lateral and septal segments with the greatest contraction delay assessed by TDI ( $\left.T_{T D I}\right)$ (Figure 2) [8, 9].

Each measurement was taken from the average of three continuous cardiac cycles. The time difference ( $T_{P W \text {-TDI }}$ ) between $T_{P W}$ and $T_{T D I}$ was used for evaluation of LVMD.

All measurements were analyzed by one observer with clinical and echocardiographic experience. To test intra- and inter-observer variability, $T_{P W}$ and $T_{T D I}$ in 20 randomly selected patients were measured again by observers $A$ and $B$, who were blinded to the previous analysis. The one observer who made all measurements was not one of the two observers used for variability assessment.

Continuous variables were presented as the mean \pm 1 standard deviation (SD) and were compared using the analysis of variance (one way ANOVA). Categorical data were assessed with the $\chi^{2}$ test or Fisher's exact test if the cell sizes were $<5$. The receiver operating characteristic (ROC) curve and accuracy matrix analysis were used for evaluation of the cutoff value of $T_{P W}$-TDI for the diagnosis of LVMD and agreement between LVMD derived by Doppler echocardiography and a positive response to CRT. The mean percentage error (difference/mean), intra-class and inter-class 


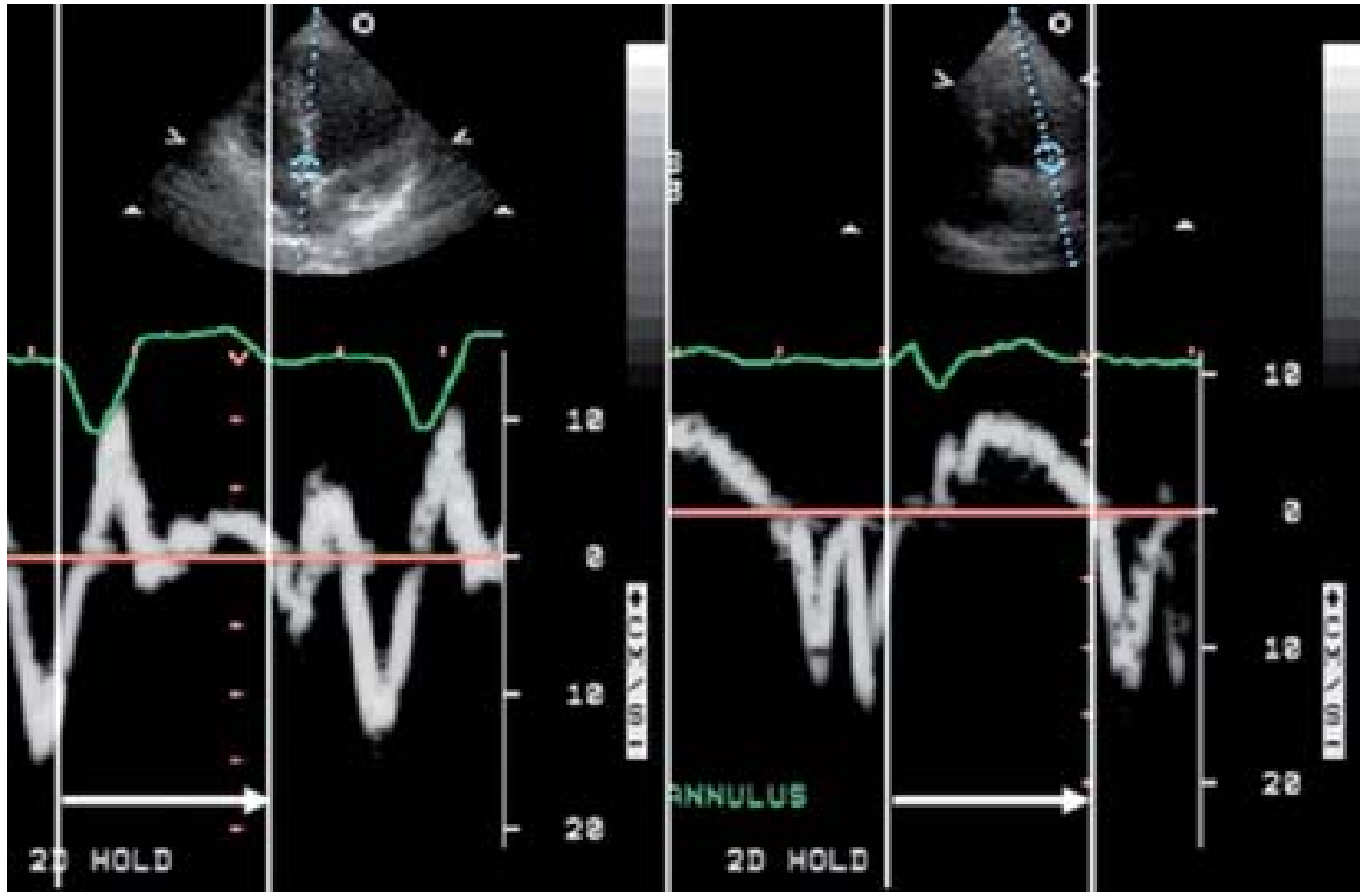

Figure 2. The time from onset of the QRS wave to the end of the systolic wave in basal segments with greatest delay (basal septal segment [left] or basal lateral wall [right] of left ventricle) assessed by TDI

regression, and Bland-Altman plot were used for evaluation of intra- and inter-observer variability. A $p$ value of $<0.05$ was considered statistically significant.

The study was approved by the Institutional Review Board of Creighton University School of Medicine.

\section{Results}

The implantation procedure was successfully performed in all 108 patients. There were no major complications after CRT implantation. The left ventricular capture threshold was $1.85 \pm 1.06$ volts, and the mean left ventricular pacing impedance was 1,004 \pm 350 ohms. The QRSD at baseline and after CRT were $165 \pm 28 \mathrm{~ms}$ and $158 \pm 30 \mathrm{~ms}$ ( $p$ not significant) during follow-up of $17 \pm 11$ months.

The patient demographics are listed in Table I. As seen in Table I, the use of $\beta$-blockers was $90 \%$ and of angiotensin-converting enzyme inhibitors or angiotensin receptor blockers was $89 \%$ in our patients. Table I also shows that $64 \%$ of the patients had coronary artery disease, $18 \%$ dilated cardiomyopathy, $14 \%$ hypertensive heart disease, and $5 \%$ valvular heart disease. In the PROSPECT Trial, $54 \%$ of the patients had coronary artery disease.

All patients underwent integrated echocardiographic measurements before CRT and at a mean duration of $15 \pm 11$ months after CRT. The
LVEF at baseline and after CRT were $20 \pm 7 \%$ and $26 \pm 13 \%, p<0.0001$. The $T_{\text {PW-TDI }}$ at baseline and after CRT were $74 \pm 48 \mathrm{~ms}$ and $49 \pm 31 \mathrm{~ms}, p<0.0001$. Forty-eight of 108 patients (44\%) had a positive response to CRT. A positive response to CRT occurred in 23 of 69 patients (33\%) with ischemic heart disease and in 25 of 39 patients (64\%) with nonischemic heart disease $(p=0.004)$. There was no significant difference in follow-up duration between

Table I. Patient demographics

\begin{tabular}{|lc|}
\hline Variable & $N(\%)$ \\
\hline Men & $78(72)$ \\
\hline Women & $30(28)$ \\
\hline Age [years] & $70 \pm 10$ \\
\hline QRS duration [ms] & $165 \pm 28$ \\
\hline Coronary artery disease & $69(64)$ \\
\hline Hypertension & $15(14)$ \\
\hline Valvular heart disease & $5(5)$ \\
\hline Dilated cardiomyopathy & $19(18)$ \\
\hline$\beta$-Blocker & $97(90)$ \\
\hline Angiotensin-converting enzyme inhibitors & $64(59)$ \\
\hline Angiotensin receptor blockers & $32(30)$ \\
\hline $\begin{array}{l}\text { Diuretics including spironolactone } \\
\text { or eplerenone }\end{array}$ & $96(89)$ \\
\hline Digoxin & $78(72)$ \\
\hline
\end{tabular}


responders to CRT (16 \pm 11 months) and nonresponders to CRT (14 \pm 11 months).

The intra-observer variability for $T_{P W}$ and $T_{T D I}$ were $1.5 \pm 0.24 \%$ and $1 \pm 0.17 \%$, respectively. There was an excellent correlation $(r=0.98$ and $r=0.99$, $p<0.001$ and $p<0.001$ ) and agreements (mean difference $6.7 \pm 10.7 \mathrm{~ms}$ and $2.8 \pm 9.1 \mathrm{~ms}$ ) in $\mathrm{T}_{\mathrm{PW}}$ and $T_{T D I}$ between the first and second measurements measured by one observer at different times, respectively (Figure 3). The inter-observer variability for $T_{P W}$ and $T_{T D I}$ were $1 \pm 0.36 \%$ and $1 \pm 0.64 \%$, respectively. There was an excellent correlation ( $r=0.89$ and $r=0.86, p<0.001$ and $p<0.001)$ and agreements in $T_{P W}$ and $T_{T D I}$ (mean difference $4 \pm 15.5 \mathrm{~ms}$ and $-1 \pm 30.4 \mathrm{~ms}$ ) between the first and second observer, respectively (Figure 4).

The ROC analysis curve compared the relationship between our technique and the gold standard (response to CRT). The cutoff point for diagnosis of LVMD was $T_{\text {PW-TDI }}>50 \mathrm{~ms}$ demonstrated by ROC analysis. There were 59 of 109 patients (55\%) with an abnormal $T_{\text {PW-TDI }}$ at baseline according to the above criteria. There was no significant difference in LVMD detection rate between patients with ischemic heart disease (34 of 69 or $49 \%$ ) and without ischemic heart disease ( 25 of 39 or $64 \%$ ). The sensitivity, specificity, and positive and negative predictive values of $\mathrm{T}_{\mathrm{PW} \text {-TDI }}>50 \mathrm{~ms}$ to predict a positive response to CRT were $98 \%, 82 \%, 81 \%$, and $98 \%$, respectively. The area under the ROC curve was 0.92 (Figure 5). There was a substantial agreement between LVMD determined by $T_{\text {PW-TDI }}$ and the positive response to CRT ( $\kappa=0.80$, proportion agreement 0.82 , and Bias Index-0.13).

Table II shows in responders versus nonresponders to CRT the mean QRSD, LVEF, and $T_{P W-T D I}$, at baseline, during short-term follow-up, and during long-term follow-up. Table II also lists levels of statistical significance.

\section{Discussion}

Tissue Doppler methods for qualitatively and quantitatively assessing intraventricular dyssynchrony are useful in selecting and monitoring patients for CRT. Using dyssynchrony indexes based on tissue velocity measurements, Yu et al. [13] and Bax et al. [14] demonstrated a high predictive value for both symptomatic improvement compared to remodeling and long-term prognosis after CRT. Aspects of acquisition and analysis that may lead to poor reproducibility have limited this technique. Poor intra- and inter-observer variability (usually $>5 \%$ ) is
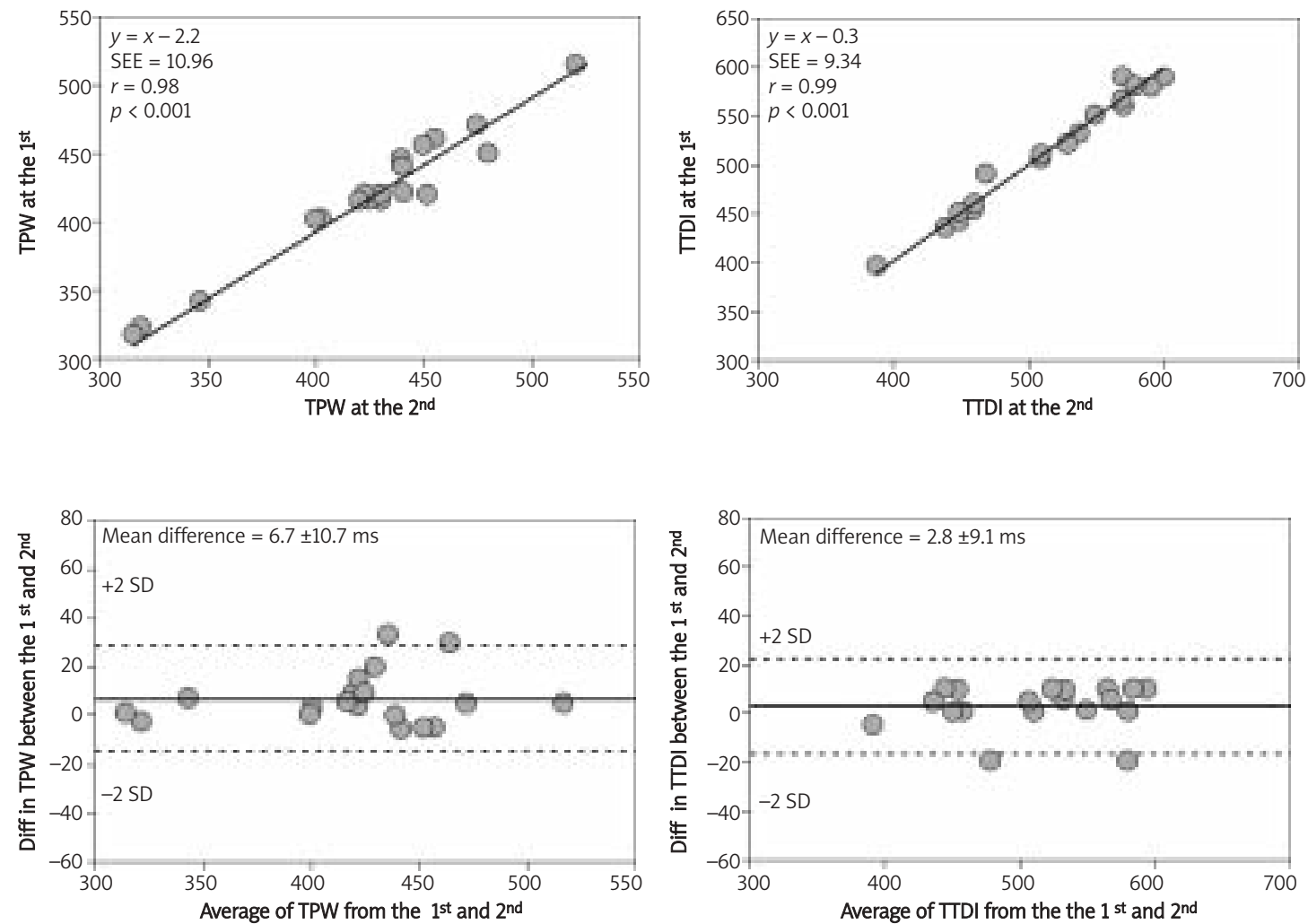

Figure 3. Intra-observer variabilities for $\mathrm{T}_{\mathrm{PW}}$ and $\mathrm{T}_{\mathrm{TDI}}$. There was excellent correlation between the first and second measurements ( $r=0.98$ [top left] and 0.99 [top right], $p<0.001$ in both). The mean difference (Diff) between first and second measurements was $6.7 \pm 10.7 \mathrm{~ms}$ (bottom left) and $2.8 \pm 9.1 \mathrm{~ms}$ (bottom right) 

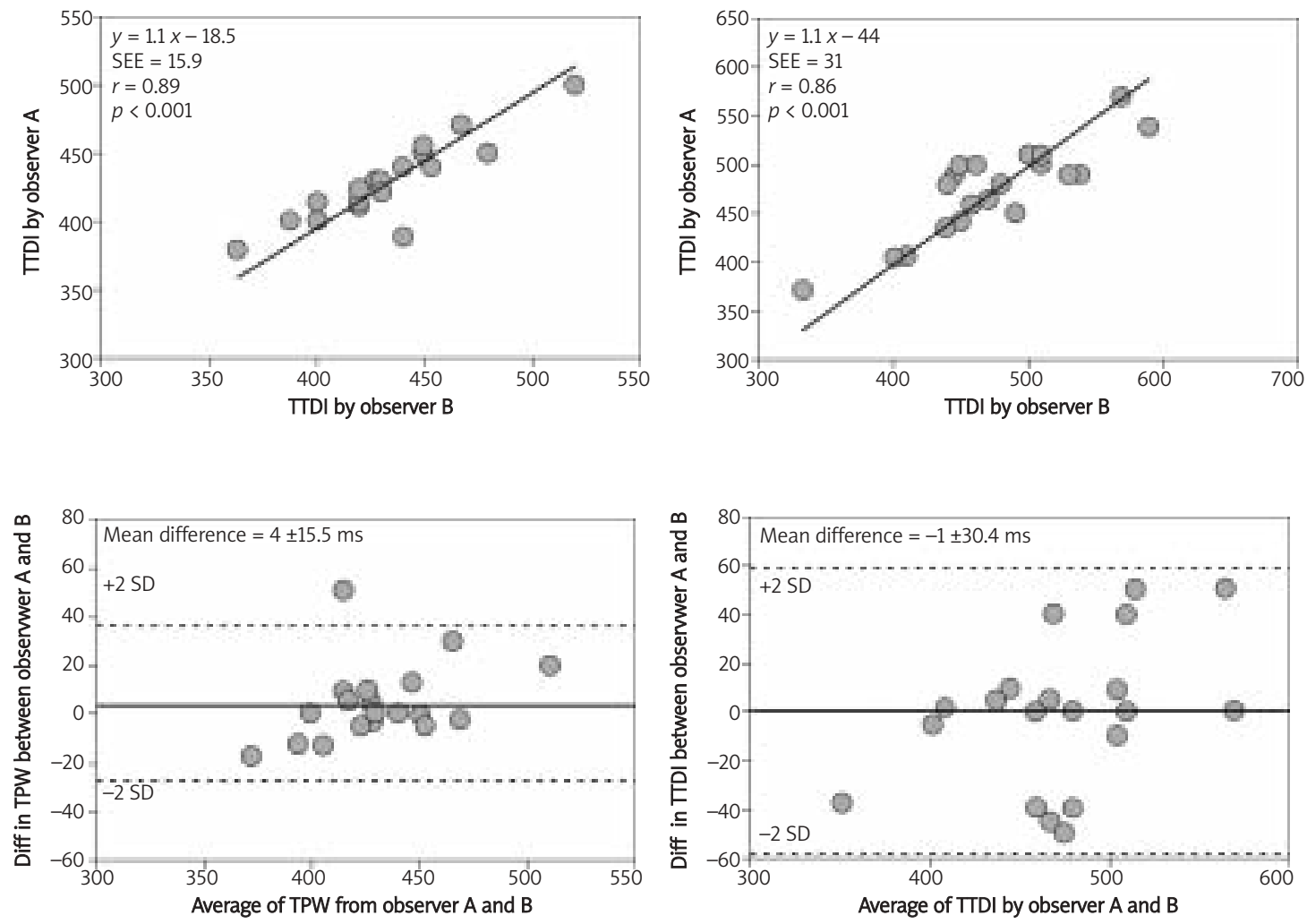

Figure 4. Inter-observer variabilities for $\mathrm{T}_{\mathrm{PW}}$ and $\mathrm{T}_{\mathrm{TDI}}$. There was excellent correlation between two observers ( $r=0.89$ [top left] and 0.86 [top right], $p<0.001$ in both). The mean difference (Diff) between observers A and B was $4 \pm 15.5 \mathrm{~ms}$ (bottom left) and $-1 \pm 30.4 \mathrm{~ms}$ (bottom right), respectively

the main limitation in evaluation of LVMD determined by TDI only, and the issue was often evaded by authors $[15,16]$. Jansen et al. [17] studied 69 patients by TDI before and after CRT. The intraobserver and inter-observer variability for measurements of the time interval from the $Q$ wave to the systolic peak velocity were $5.3 \pm 3.4 \%$ and 7.1 $\pm 6.6 \%$, respectively. These results are not satisfactory for clinical use in prediction of a positive response to CRT before biventricular pacemaker implantation. Moreover, the recently finished Predictors of Response to CRT (PROSPECT) trial found that the ability of the 12 echocardiographic parameters to predict clinical composite score response varied widely, with sensitivity ranging from $6 \%$ to $74 \%$ and specificity from $31 \%$ to $93 \%$ [18]. The investigators pointed out that no single echocardiographic measure of dyssynchrony may be recommended to improve patient selection for CRT beyond current guidelines [18]. The intra- and inter-observer variability in the present study was improved compared to the study by Jansen et al. [17].

We agree with the data reported in the PROSPECT trial using the 12 echocardiographic parameters studied [18]. However, the PROSPECT trial did not investigate the method used by Perez de Isla et al. [9]. Our study using this method

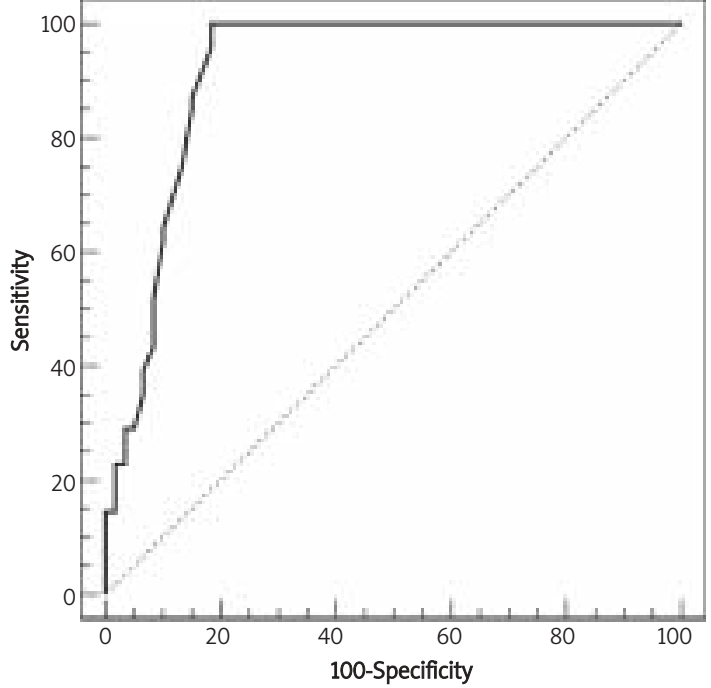

Figure 5. When a positive response to CRT was defined as left ventricular systolic volume reduction $\geq 15 \%$ after CRT, the area under the ROC curve was 0.92

showed an area under the ROC curve of 0.92 (Figure 5) compared to 0.62 for the PROSPECT trial. Our intra-observer variability and inter-observer variability were also better than the values reported in the PROSPECT trial. A major limitation of our 
Table II. QRS duration, $T_{P W-T D I}$, and left ventricular ejection fraction (LVEF) at baseline and during short-term and long-term follow-up

\begin{tabular}{|c|c|c|c|c|c|c|}
\hline & & Baseline & Short-term & Value of $p$ & Long-term & Value of $p$ \\
\hline \multirow[t]{3}{*}{ QRSD [ms] } & Responders & $168 \pm 23$ & $150 \pm 25$ & 0.03 & $140 \pm 31$ & $0.003^{*}$ \\
\hline & Non-responders & $162 \pm 30$ & $158 \pm 35$ & NS & $163 \pm 36$ & NS \\
\hline & Value of $p$ & NS & NS & & 0.04 & \\
\hline \multirow[t]{3}{*}{ TPW-TDI [ms] } & Responders & $112 \pm 46$ & $43 \pm 30$ & $<0.0001$ & $43 \pm 22$ & $<0.0001^{\star}$ \\
\hline & Non-responders & $54 \pm 37$ & $45 \pm 28$ & NS & $61 \pm 38$ & NS \\
\hline & Value of $p$ & $<0.0001$ & NS & & NS & \\
\hline \multirow[t]{3}{*}{ LVEF [\%] } & Responders & $21 \pm 7$ & $33 \pm 14$ & 0.002 & $32 \pm 16$ & $0.009^{\star}$ \\
\hline & Non-responders & $19 \pm 7$ & $20 \pm 9$ & NS & $24 \pm 9$ & NS \\
\hline & Value of $p$ & NS & 0.002 & & NS & \\
\hline
\end{tabular}

${ }^{*}$ Compared to baseline, NS - not significant

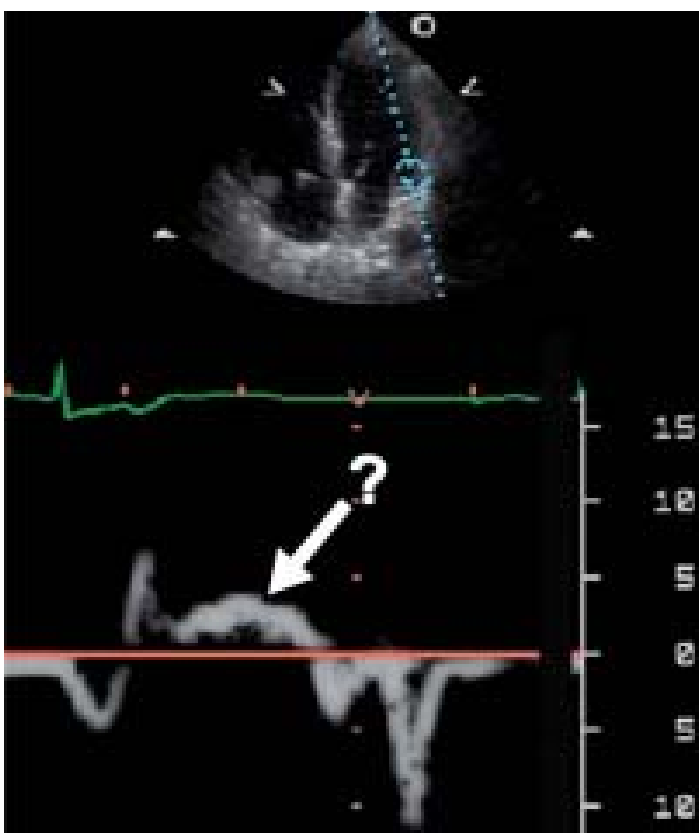

Figure 6. Wall motion velocity spectrum by tissue Doppler imaging. It is difficult to define the end point of peak velocity because of its blunt nature

study and of the PROSPECT trial is that scar tissue was not assessed because scar tissue cannot be evaluated by echocardiography.

Our study did not investigate the effect of CRT on symptoms, which are a subjective endpoint. Our positive response to CRT of $44 \%$ is low but consistent with other studies [12, 13].

Perez de Isla et al. [9] successfully detected LVMD by the method of combined PW and TDI in 193 heart failure patients. However, they did not perform a validation study using combined $\mathrm{PW}$ and TDI to predict a positive response to CRT. Our proposed study using Isla's method demonstrated that the sensitivity, specificity, and agreement were close to the method using Bax's method, but the intra- and inter-observer reproducibility improved significantly from $5-7 \%$ to $1-1.5 \%$. The reason for the improvement is that Perez de Isla et al. measured the time interval from the $\mathrm{Q}$ wave to the end of left ventricular ejection by TDI instead of the time interval measured from the $\mathrm{Q}$ wave to the peak velocity because the peak velocity profile has a blunt nature and double peaks (Figure 6).

The time derived by PW represents the mean time interval of left ventricular ejection, and the time derived by TDI represents the segmental time delay of left ventricular ejection. It easily defines the end point from left ventricular flow and motion velocity spectrum by PW and TDI (Figures $1,2)$, as compared to a method which defines the end point of peak systolic velocity spectrum by TDI only.

The detection rate of LVMD is lower both in Perez de Isla's et al. study (39\%) and our current study (55\%) in patients with heart failure and a wide QRSD compared to other studies (60-75\%) [13]. However, the lower LVMD detection rate did not affect the sensitivity for prediction of a positive response to CRT in our study. We found that the lower LVMD detection rate (55\%) was consistent with a lower CRT response rate (44\%), and the cause of a lower CRT response rate may be related to ischemic heart disease. Previous studies suggested that CRT may be less beneficial among heart failure patients with ischemic heart disease compared to non-ischemic heart disease [19-22].

Although the follow-up duration in this study was not constant for each patient after CRT, there was no significant difference in follow-up duration between responders and non-responders. Prospective studies using a large number of patients with longer followup times are warranted.

In conclusion, left ventricular dyssynchrony detected by the method of combined PW and TDI demonstrated a high reproducibility, sensitivity, specificity, positive and negative predictive values, and agreement with a positive response to CRT compared to single echocardiographic measurements of left ventricular dyssynchrony described previously [14, 23, 24]. 


\section{Acknowledgments}

We are extremely grateful to Mr. Tim Farkas and Mr. Curt Estell for their great help in data collection.

\section{References}

1. Abraham WT, Fisher WG, Smith AL, et al. Cardiac resynchronization in chronic heart failure. N Engl J Med 2002; 346: 1845-53.

2. Cleland JG, Daubert JC, Erdmann E, et al. Cardiac Resynchronization-Heart Failure (CARE-HF) Study Investigators. The effect of cardiac resynchronization on morbidity and mortality in heart failure. N Engl J Med 2005; 352: 1539-49.

3. Gras D, Leclercq C, Tang AS, et al. Cardiac resynchronization therapy in advanced heart failure: the multicenter InSync clinical study. Eur J Heart Fail 2002; 4: 311-20.

4. Young JB, Abraham WT, Smith AL, et al. Combined cardiac resynchronization and implantable cardioversion defibrillation in advanced chronic heart failure: the MIRACLE ICD Trial. JAMA 2003; 289: 2685-94.

5. Linde C, Braunschweig F, Gadler F, Bailleul C, Daubert JC. Long-term improvements in quality of life by biventricular pacing in patients with chronic heart failure: results from the Multisite Stimulation in Cardiomyopathy Study (MUSTIC). Am J Cardiol 2003; 91: 1090-5.

6. Anderson LJ, Miyazaki C, Sutherland GR, Oh JK. Patient selection and echocardiographic assessment of dyssynchrony in cardiac resynchronization therapy. Circulation 2008; 117: 2009-23.

7. Rosanio S, Schwarz ER, Ahmad M, et al. Benefits, Unresolved questions, and technical issue of cardiac resynchronization therapy for heart failure. Am J Cardiol 2005; 96: 710-7.

8. Perez de Isla L, Ortiz Oficialdegui P, Florit J, Angel GarciaFernandez M, Sanchez V, Zamorano J. Usefulness of clinical, electrocardiographic, and echocardiographic parameters to detect cardiac asynchrony in patients with left ventricular dysfunction secondary to Ischemic or nonischemic heart disease. J Am Soc Echocardiogr 2006; 19: 1338-44.

9. Pérez de Isla L, Florit J, Garcia-Fernandez MA, Evangelista A, Zamorano J; RAVE (Registro de Asincronía Ventricular en España-Spanish Ventricular Asynchrony Registry) study investigators. Prevalence of echocardiographically detected ventricular asynchrony in patients with left ventricular systolic dysfunction. J Am Soc Echocardiogr 2005; 18: 850-9.

10. Hunt SA, Abraham WT, Chin MH, et al. ACC/AHA 2005 Guideline Update for the diagnosis and management of chronic heart failure in the adult. Circulation 2005; 112: 1825-52.

11. Lang RM, Bierig M, Devereux RB, et al. Recommendations for Chamber Quantification: A Report from the American Society of Echocardiography's Guidelines and Standards Committee and the Chamber Quantification Writing Group. Developed in Conjunction with the European Association of Echocardiography, a branch of the European Society of Cardiology. J Am Society Echocardiogr 2005; 18: 1440-63.

12. Pitzalis MV, Iacoviello M, Romito R, et al. Cardiac resynchronization therapy tailored by echocardiographic evaluation of ventricular asynchrony. J Am Coll Cardiol 2002; 40: 1615-22.
13. Yu CM, Fung JW, Zhang Q, et al. Tissue Doppler imaging is superior to strain rate imaging and postsystolic shortening on the prediction of reverse remodeling in both ischemic and nonischemic heart failure after cardiac resynchronization therapy. Circulation 2004; 110: 66-73.

14. Bax JJ, Bleeker GB, Marwick TH, et al. Left ventricular dyssynchrony predicts response and prognosis after cardiac resynchronization therapy. J Am Coll Cardiol 2004; 44: 1834-40.

15. Penicka M, Bartunek J, De Bruyne B, et al. Improvement of left ventricular function after cardiac resynchronization therapy is predicted by tissue Doppler imaging echocardiography. Circulation 2004; 109: 978-83.

16. Sogaard P, Egeblad H, Pedersen AK, et al. Sequential versus simultaneous biventricular resynchronization for severe heart failure: evaluation by tissue Doppler imaging. Circulation 2002; 106: 2078-84.

17. Jansen AH, Bracke F, van Dantzig JM, et al. Optimization of pulsed wave tissue Doppler to predict left ventricular reverse remodeling after cardiac resynchronization therapy. J Am Soc Echocardiogr 2006; 19: 185-91.

18. Chung ES, Leon AR, Tavazzi L, et al. Results of the Predictors of Response to CRT (PROSPECT) Trial. Circulation 2008; 117: 2608-16.

19. Gasparini M, Mantica M, Galimberti P, et al. Is the outcome of cardiac resynchronization therapy related to the underlying etiology? Pacing Clin Electrophysiol 2003; 26: $175-80$.

20. Sutton MG, Plappert T, Abraham WT, et al. Effect of cardiac resynchronization therapy on left ventricular size and function in chronic heart failure. Circulation 2003; 107: 1985-90.

21. Birnie DH, Tang AS. The problem of non-response to cardiac resynchronization therapy. Curr Opinion Cardiol 2006; 21: 20-6.

22. Bleeker GB, Kaandorp TAM, Lamb HJ, et al. Effect of posterolateral scar tissue on clinical and echocardiographic improvement after cardiac resynchronization therapy. Circulation 2006; 113: 969-76.

23. Rajdev A, Aronow WS, Lai HM, et al. Comparison of left ventricular ejection fraction by single photon computed tomographic myocardial perfusion imaging versus coronary computed tomography angiography. Arch Med Sci 2009; 5: 28-31.

24. Cronin A, Aronow WS, Devabhakturi S, et al. Prevalence of incidental noncardiac findings diagnosed by computer tomography in 875 consecutive patients in an academic outpatient cardiac computer tomography facility. Arch Med Sci 2008; 4: 401-3. 